\title{
Floristic, structure and ecological groups of a tree community in the Atlantic Rainforest at Serra do Mar, SP, Brazil
}

\author{
Vitor de Andrade KAMIMURA ${ }^{1 *}$, Rodrigo Ferreira MORAIS ${ }^{2}$, Carlos Alfredo JOLY ${ }^{3}$, \\ Marco Antonio ASSIS ${ }^{1}$
}

\author{
${ }^{1}$ Departamento de Botânica, Universidade Estadual Paulista Júlio de Mesquita Filho, Rio Claro, São Paulo, Brasil. \\ ${ }^{2}$ Centro de Ciências Naturais, Universidade do Estado do Piauí, Teresina, Piauí, Brasil. \\ ${ }^{3}$ Departamento de Biologia Vegetal, Universidade Estadual de Campinas, Campinas, São Paulo, Brasil. \\ *E-mail: vitorkami@msn.com
}

Recebido em março/2018; Aceito em julho/2018.

\begin{abstract}
The present study evaluated the floristic and structural composition of a tree community in a Lowland Atlantic Rainforest along a forest continuum in Brazilian southeast, assessing the species distribution among ecological groups. For this purpose, we sampled all trees with $\mathrm{PBH} \geq 15 \mathrm{~cm}$, including palm trees and tree ferns, in a total area of one hectare, divided into subplots of $10 \times 10 \mathrm{~m}$. The studied community was also analyzed through the Shannon diversity index and Pielou equability, and its species distribution among dispersal syndromes and successional classes. We recorded 1,120 living individuals distributed in 131 species of 40 families. The richest families were Myrtaceae (32 species), Fabaceae (12) and Rubiaceae (11), and Euterpe edulis was the most abundant species (11.1\% of the total). In the study area, there were found four species on the Livro Vermelho da Flora do Brasil and recorded a dominance of zoochoric species ( $86.7 \%$ of species classified) and non-pioneers (71.6\%). The general results of the community structure and species composition were similar with other surveys carried out in the same region of this study. Finally, our results corroborate the great diversity of trees and a dominance of zoochoric and non-pioneer species in the Atlantic Rainforest continuous.
\end{abstract}

Keywords: dispersal syndromes, diversity, ombrophilous dense forest, successional groups.

\section{Florística, estrutura e grupos ecológicos de uma comunidade arbórea na Floresta Atlântica, Serra do Mar, SP, Brasil}

RESUMO: Neste trabalho, avaliamos a composição florística e estrutural de uma comunidade arbórea em um contínuo da Floresta Atlântica das Terras Baixas no sudeste brasileiro, acessando a distribuição das espécies entre grupos ecológicos. Para tanto, foram amostrados todos os indivíduos arbóreos com $\mathrm{PAP} \geq 15 \mathrm{~cm}$, incluindo palmeiras e samambaias arborescentes, em um hectare, dividido em sub-parcelas de 10x10 m. A comunidade foi analisada por meio do índice de diversidade de Shannon e equabilidade de Pielou, e distribuição de suas espécies entre síndromes de dispersão e classes sucessionais. Registramos 1.120 indivíduos vivos distribuídos em 133 espécies de 41 famílias. As famílias mais ricas em espécies foram Myrtaceae (32 espécies), Fabaceae (12) e Rubiaceae (11), e Euterpe edulis a espécie mais abundante (11,1\% do total). Na área de estudo, foram encontradas quatro espécies na lista espécies ameaçadas do Livro Vermelho da Flora do Brasil, e registrada uma dominância de espécies Zoocóricas (86,7\% das espécies classificadas) e Não Pioneiras (73,5\%). Os resultados gerais da estrutura e composição de espécies da comunidade foram similares à de outros levantamentos realizados na mesma região deste estudo. Por fim, nossos resultados corroboram a grande diversidade arbórea e uma dominância de espécies Zoocóricas e Não-Pioneiras em contínuos de Floresta Atlântica.

Palavras-chave: classe sucessional, floresta ombrófila densa, diversidade, síndrome de dispersão.

\section{INTRODUCTION}

Tropical Forests, especially those located in the Neotropical region, are characterized by a great biological diversity and high degree of endemism (LEIGH et al., 2004; MITTERMEIER et al., 2011). In this regard, the Atlantic Forest has a high floristic richness, presenting more than 15,004 vascular species, of which $49.5 \%$ are exclusive to the phytogeographic domain (BFG, 2015). This phytogeographic domain has been established in the Brazilian territory about 70 million years ago, and it is undoubtedly the oldest forest formation in the country (FIASCHI; PIRANI, 2009).

In general, the Atlantic Forest domain presents two main vegetation types (MORELLATO; HADDAD, 2000), the Seasonal Semideciduous Forest and the Ombrophilous Dense
Forest. Due to more pronounced processes of fragmentation and deforestation in areas with high anthropogenic pressure (LAURANCE, 2009; TURNER, 1996), as observed in the coastal regions of Brazil, now has only remained $11 \%$ of its original forest cover, and a large part of the remnant is situated in southeastern Brazil (RIBEIRO et al., 2009).

Among the Neotropical Forests, the Atlantic Rainforest is considered one of the most threatened hotspots (LAURANCE, 2009; MYERS et al., 2000). Although, the species composition studies and structural surveys of the Atlantic Rainforest has been highly developed in the last two decades (e.g. OLIVEIRA-FILHO; FONTES 2000; TABARELLI; MANTOVANI 1999), remains a demand to new surveys for comprehend the complexity and diversity of this biome 
(EISENLOHR; OLIVEIRA-FILHO, 2015; SAITER et al., 2015). Hence, there is also a lack of studies for tree communities in certain physiognomies of the Brazilian southeast, mainly, in areas that have undergone an exhaustive human occupation, as Lowland Atlantic Rainforest. (EISENLOHR; OLIVEIRA-FILHO, 2015; JOLY et al., 2012; LAURANCE, 2009; OLIVEIRA-FILHO; FONTES, 2000; SANCHEZ et al., 2013).

The floristic and phytosociological surveys are pointed out as effective tools to describe tree communities and analyze the conservation status of vegetation (EISENLOHR; OLIVEIRAFILHO, 2015; JOLY et al., 2012). This type of studies, associated with the assessment of ecological groups, can provide an improvement in the understanding about the communities' structure and species distribution (TABARELLI; PERES, 2002; WIJDEVEN; KUZEE, 2000).

Thus, we assessed the floristic composition and structure of a preserved tree community in the Lowland Atlantic Rainforest of southeastern Brazil. Furthermore, we described the tree community by species distribution among ecological groups (dispersal syndrome and successional categories) and the contribution of each group in the community structure.

\section{MATERIALS AND METHODS}

\subsection{Study area}

This study was conducted in one hectare of Atlantic Rainforest in the Núcleo Picinguaba, Parque Estadual da Serra do Mar, Ubatuba, Brazil (Figure 1). The plot surveyed is located in the Lowland Atlantic Rainforest according to the IBGE (2012), at coordinates $23^{\circ} 20^{\prime} 8^{\prime \prime} \mathrm{S} 44^{\circ} 50^{\prime} 4$ "W between 41 and 63 meters of altitude. The climate is tropical rainforest (AF according to the Koppen system, 1948) with a mean value of annual rainfall exceeding $2,200 \mathrm{~mm}$.

The plot surveyed $(100 \times 100 \mathrm{~m})$ integrates a sample (plot "C") of a project entitled, "Floristic composition, structure and functioning of the Atlantic Rainforest of Picinguaba and Santa Virginia Nucleus in Serra do Mar State Park." In total, the project sampled 14 ha along an altitudinal gradient in the Atlantic Rainforest (JOLY et al., 2012). The plots were divided into 100 subplots $(10 \times 10 \mathrm{~m})$, georeferenced and delimited with PVC stakes.

\subsection{Sampling and ecological classification}

We sampled all trees with PBH (perimeter at breast height) $\geq 15 \mathrm{~cm}$, including palm trees and tree ferns, per the protocol established by Joly; Martinelli (2008). The materials were identified using specialized bibliographies and, when possible, by consulting taxonomic specialists. The botanic material was deposited in the collections of Herbaria UEC, IAC, and HRCB. The distribution of taxa by families followed the proposal of the Angiosperm Phylogeny Group IV (APG, 2016). For phytosociological analysis, we recorded the PBH values and the height of each tree individual sampled. The categorization of species in ecological groups was obtained using specific literature (CATHARINO et al. 2006; GRESSLER et al. 2006; SÃO PAULO, 2008) and the analysis of diasporas when collected. The species were classified in the dispersal syndromes according to the Pijl classification (VAN DER PIJL, 1982): autochory, anemochory and zoochory, and successional categories: pioneer latosensu and non-pioneer latosensu according to Whitmore (1989). In addition, we assessed the threatened status of species sampled, according to
Livro Vermelho da Flora do Brasil (MARTINELLI; MORAES, 2013).

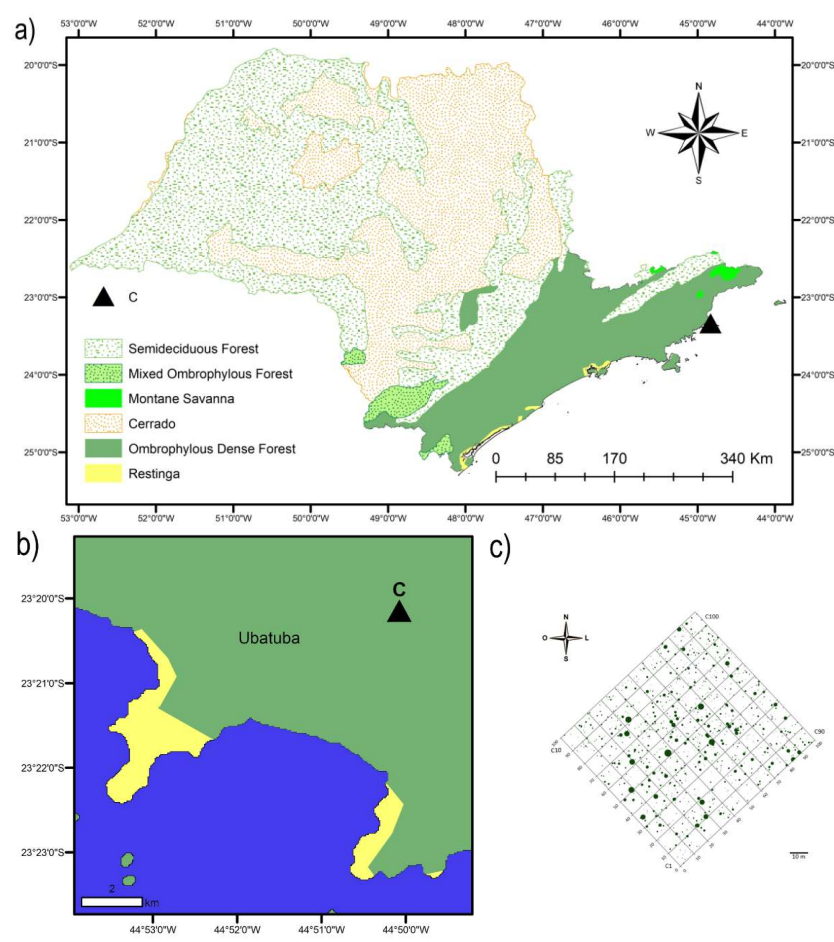

Figure 1. São Paulo vegetation map (a), plot location (b) and individual distributions (c) of tree communities surveyed in the Atlantic Rainforest of Serra do Mar State Park. Ubatuba, Brazil. CPlot surveyed (tree community) in this work. - - The circle size corresponds to the individual basal area.

Figura 1. Mapa da vegetação de São Paulo (a), localização do plot (b) e distribuição dos indivíduos (c) da comunidade arbórea analisada na Floresta Atlântica do Parque Estadual da Serra do Mar, Ubatuba, Brasil. Parcela C - Parcela levantada (comunidade arbórea) este trabalho. • - O tamanho do círculo corresponde a área basal do indivíduo.

\subsection{Data analysis}

Phytosociological parameters such as relative density, frequency, and dominance, and the importance value index were calculated according to Mueller-Dombois; Ellenberg (1974). We estimated the Shannon diversity index $\left(\mathrm{H}^{\prime}\right)$ and Pielou equability $\left(\mathrm{J}^{\prime}\right)$, and we also conducted a rarefaction curve to assess the sample sufficiency by species accumulation (MORO; MARTINS, 2011). We also evaluated the structure parameters among ecological groups considering the species richness, abundance, dominance and mean values of height and diameter of each group. The analyses were performed using R software (R DEVELOPMENT CORE TEAM, 2017).

\section{RESULTS}

We surveyed 1,120 living individuals and 124 standing stems dead, which were excluded from the analyses of ecological groups. A total of 131 species were sampled in 40 families, with six individuals remaining indeterminate (Table $1)$. We found four sampled species in the list of threatened species of the Livro Vermelho da Flora do Brasil (MARTINELI; MORAES, 2013), two in the Vulnerable status, Cupania furfuracea Radkl. (Sapindaceae) and Euterpe edulis Mart. (Arecaceae), and two in the Endangered status, Eugenia magnibracteolata Mattos e D. Legrand (Myrtaceae) and Virola bicuhyba (Schott ex Spreng.) Warb. 
(Myristicaceae). The Shannon diversity was 3.91 nats/ind and the Pielou equability was 0.81 .

Table 1. Species list, structural parameters, dispersal syndromes and successional class of surveyed community in the Atlantic Rainforest of Serra do Mar State Park, São Paulo, Brazil, (Abd - Abundance; RelFr - Relative Frequency; RelDo- Relative Dominance. IV - Importance Value; Zoo - Zoochory; Ane - Anemochory; Aut - Autochory. NP - Non-Pioneer. P - Pioneer. NC - Not-Classified.

Tabela 1. Relação das espécies, descritores estruturais, síndromes de dispersão e classe sucessional, da comunidade levantada na Floresta Atlântica do Parque Estadual da Serra do Mar. (Abd. - Abundância; RelFr - Frequência relativa; RelDo - Dominância Relativa; IV - Valor de Importância; Zoo - Zoocórica; Ane - Anemocórica; Aut - Autocórica; NP - Não Pioneira; P - Pioneira; NC - Não Classificadas).

\begin{tabular}{|c|c|c|c|c|c|c|c|}
\hline Family & Specie & Abd. & RelFR & RelDo & IV & $\begin{array}{c}\text { Dispersion } \\
\text { syndrome }\end{array}$ & $\begin{array}{c}\text { Successional } \\
\text { Class }\end{array}$ \\
\hline \multirow{2}{*}{ Annonaceae } & Guatteria australis A.St.-Hil. & 4 & 0.45 & 0.21 & 0.33 & Zoo & NP \\
\hline & Annonaceae 1 & 3 & 0.35 & 0.25 & 0.29 & $\mathrm{NC}$ & $\mathrm{NC}$ \\
\hline \multirow[t]{2}{*}{ Araliaceae } & Schefflera angustissima Marchal (Frodim) & 8 & 0.92 & 0.35 & 0.66 & Zoo & $\mathrm{P}$ \\
\hline & Schefflera calva (Cham.) Frodin e Fiaschi & 1 & 0.12 & 0.06 & 0.09 & Zoo & $\mathrm{P}$ \\
\hline \multirow[t]{3}{*}{ Arecaceae } & Astrocaryum aculeatissimum (Schott) Burret & 7 & 0.69 & 0.24 & 0.52 & Zoo & NP \\
\hline & Euterpe edulis Mart. & 124 & 7.04 & 2.7 & 6.95 & Zoo & NP \\
\hline & Syagrus pseudococos (Raddi) Glassman & 19 & 1.85 & 1.89 & 1.83 & Zoo & NP \\
\hline Bignoniaceae & Jacaranda puberula Cham. & 2 & 0.23 & 0.14 & 0.18 & Ane & $\mathrm{P}$ \\
\hline \multirow{2}{*}{ Boraginaceae } & Cordia silvestris Fresen. & 1 & 0.12 & 0.14 & 0.11 & Zoo & NP \\
\hline & Cordia taguahyensis Vell. & 10 & 0.92 & 0.68 & 0.83 & Zoo & NP \\
\hline Cardiopteridaceae & Citronella paniculata (Mart.) R.A. Howard & 10 & 1.04 & 0.21 & 0.71 & Zoo & $\mathrm{P}$ \\
\hline Caricaceae & Jacaratia heptaphylla (Vell.) A. DC. & 1 & 0.12 & 0.18 & 0.13 & Zoo & NP \\
\hline Celastraceae & Salacia grandifolia (Mart.) G. Don & 3 & 0.35 & 0.04 & 0.22 & Zoo & NP \\
\hline \multirow[t]{2}{*}{ Chrysobalanaceae } & Couepia venosa Prance & 9 & 0.92 & 2.24 & 1.33 & Zoo & NP \\
\hline & Hirtella hebeclada Moric. Ex DC. & 1 & 0.12 & 0.04 & 0.08 & Zoo & NP \\
\hline Clusiaceae & Garcinia gardneriana (Planch. e Triana) Zappi & 17 & 1.62 & 0.81 & 1.32 & Zoo & NP \\
\hline \multirow[t]{2}{*}{ Cyatheaceae } & Alsophila sternbergii (Sternb.) D.S. Conant & 23 & 1.62 & 1.64 & 1.70 & Ane & $\mathrm{P}$ \\
\hline & Cyathea phalerata Mart. & 5 & 0.46 & 0.24 & 0.38 & Ane & $\mathrm{P}$ \\
\hline Elaeocarpaceae & Sloanea guianensis (Aubl.) Benth. & 11 & 1.27 & 0.91 & 1.06 & Zoo & NP \\
\hline \multirow{7}{*}{ Euphorbiaceae } & Alchornea glandulosa Poepp. & 6 & 0.58 & 0.54 & 0.55 & Zoo & $\mathrm{P}$ \\
\hline & Alchornea triplinervia (Spreng.) Mull. Arg. & 1 & 0.12 & 0.11 & 0.11 & Zoo & $\mathrm{P}$ \\
\hline & Mabea piriri Aubl. & 16 & 1.27 & 1.73 & 1.48 & Aut & $P$ \\
\hline & Pausandra morisiana (Casar.) Radlk. & 2 & 0.23 & 0.02 & 0.14 & Zoo & NP \\
\hline & Sapium glandulosum (L.) Morong & 2 & 0.23 & 0.77 & 0.39 & Zoo & $\mathrm{P}$ \\
\hline & Tetrorchidium rubrivenium Poepp. & 1 & 0.12 & 0.08 & 0.10 & Aut & $\mathrm{P}$ \\
\hline & Euphorbiaceae 1 & 4 & 0.46 & 0.21 & 0.34 & $\mathrm{NC}$ & $\mathrm{NC}$ \\
\hline \multirow[t]{12}{*}{ Fabaceae } & Copaifera langsdorffii Desf. & 3 & 0.35 & 0.15 & 0.26 & Zoo & NP \\
\hline & Dahlstedtia pinnata (Benth.) Malme & 3 & 0.23 & 0.03 & 0.18 & Ane & $\mathrm{P}$ \\
\hline & Inga striata Benth. & 1 & 0.12 & 0.03 & 0.08 & Zoo & $\mathrm{P}$ \\
\hline & Inga sp.1 & 3 & 0.35 & 2.13 & 0.92 & Zoo & $\mathrm{P}$ \\
\hline & Inga sp. 2 & 1 & 0.12 & 1.90 & 0.70 & Zoo & $\mathrm{P}$ \\
\hline & Lonchocarpus cultratus (Vell.) Az.Tozzi e H.C.Lima & 1 & 0.12 & 0.01 & 0.07 & Ane & $\mathrm{P}$ \\
\hline & Swartzia oblata R.S. Cowan & 6 & 0.58 & 2.25 & 1.12 & Zoo & NP \\
\hline & Swartzia simplex var. grandiflora (Raddi) R.S. Cowan & 15 & 1.50 & 1.27 & 1.39 & Zoo & NP \\
\hline & Zollernia ilicifolia (Brongn.) Vogel & 11 & 1.04 & 1.06 & 1.03 & Zoo & NP \\
\hline & Mimosoideae 1 & 1 & 0.12 & 0.31 & 0.17 & $\mathrm{NC}$ & $\mathrm{NC}$ \\
\hline & Fabaceae 1 & 3 & 0.35 & 1.37 & 0.66 & $\mathrm{NC}$ & $\mathrm{NC}$ \\
\hline & Fabaceae 2 & 1 & 0.12 & 0.40 & 0.20 & $\mathrm{NC}$ & $\mathrm{NC}$ \\
\hline \multirow[t]{6}{*}{ Lauraceae } & Cryptocaria mandiocana Meisr & 4 & 0.46 & 1.23 & 0.68 & Zoo & NP \\
\hline & Cryptocaria saligna $\mathrm{Mez}$ & 7 & 0.69 & 0.50 & 0.60 & Zoo & NP \\
\hline & Endlicheria paniculata (Spreng.) J.F. Macbr. & 9 & 0.35 & 0.12 & 0.42 & Zoo & NP \\
\hline & Ocotea dispersa (Nees) Mez & 9 & 1.04 & 0.62 & 0.82 & Zoo & NP \\
\hline & Ocotea divaricata (Nees) Mez & 1 & 0.12 & 0.14 & 0.11 & Zoo & NP \\
\hline & Ocotea teleiandra (Meisn.) Mez & 1 & 0.12 & 0.04 & 0.08 & Zoo & NP \\
\hline Lecythidaceae & Cariniana estrelensis (Raddi) Kuntze & 2 & 0.23 & 0.04 & 0.15 & Ane & NP \\
\hline \multirow[t]{2}{*}{ Malvaceae } & Eriotheca pentaphylla (Vell.) A. Robyns & 19 & 1.73 & 4.51 & 2.67 & Ane & $\mathrm{P}$ \\
\hline & Quararibea turbinata (Sw.) Poir. & 6 & 0.69 & 0.10 & 0.44 & Zoo & NP \\
\hline Melastomataceae & Miconia sp. & 2 & 0.23 & 0.08 & 0.16 & $\mathrm{NC}$ & $\mathrm{NC}$ \\
\hline \multirow[t]{3}{*}{ Meliaceae } & Guarea macrophylla Vahl & 3 & 0.35 & 0.27 & 0.29 & Zoo & $\mathrm{P}$ \\
\hline & Trichilia silvatica C. DC. & 4 & 0.46 & 0.76 & 0.53 & Zoo & NP \\
\hline & Meliaceae 1 & 1 & 0.12 & 0.02 & 0.08 & $\mathrm{NC}$ & $\mathrm{NC}$ \\
\hline \multirow[t]{4}{*}{ Monimiaceae } & Mollinedia engleriana Perkins & 1 & 0.12 & 0.02 & 0.07 & Zoo & NP \\
\hline & Mollinedia lamprophylla Perkins & 8 & 0.69 & 2.79 & 1.40 & Zoo & NP \\
\hline & Mollinedia schottiana (Spreng.) Perkins & 93 & 6.93 & 2.41 & 5.88 & Zoo & NP \\
\hline & Mollinedia sp. & 1 & 0.12 & 0.01 & 0.07 & $\mathrm{NC}$ & $\mathrm{NC}$ \\
\hline \multirow[t]{4}{*}{ Moraceae } & Ficus luschnathiana (Miq.) Miq. & 1 & 0.12 & 0.72 & 0.31 & Zoo & NP \\
\hline & Ficus pulchella Schott & 1 & 0.12 & 0.68 & 0.30 & Zoo & NP \\
\hline & Ficus sp. & 1 & 0.12 & 0.01 & 0.07 & $\mathrm{NC}$ & $\mathrm{NC}$ \\
\hline & Sorocea hilarii Gaud. & 4 & 0.35 & 0.05 & 0.25 & Zoo & NP \\
\hline Myristicaceae & Virola bicuhyba (Schott ex Spreng.) Warb. & 16 & 1.73 & 8.79 & 3.98 & Zoo & NP \\
\hline Myrtaceae & Calyptranthes lucida Mart. ex DC. & 2 & 0.23 & 0.13 & 0.18 & Zoo & NP \\
\hline
\end{tabular}


Kamimura et al.

\begin{tabular}{|c|c|c|c|c|c|c|c|}
\hline Family & Specie & Abd. & RelFR & RelDo & IV & $\begin{array}{c}\text { Dispersion } \\
\text { syndrome }\end{array}$ & $\begin{array}{c}\text { Successional } \\
\text { Class }\end{array}$ \\
\hline & Calyptranthes strigipes $\mathrm{O}$. Berg & 1 & 0.12 & 0.01 & 0.07 & Zoo & NP \\
\hline & Campomanesia guaviroba (DC) & 1 & 0.12 & 0.10 & 0.10 & Zoo & $\mathrm{P}$ \\
\hline & Campomanesia xanthocarpa O.Berg & 3 & 0.35 & 0.20 & 0.27 & Zoo & NP \\
\hline & Campomanesia sp. & 1 & 0.12 & 0.17 & 0.12 & Zoo & NP \\
\hline & Eugenia brevistyla D.Legrand & 5 & 0.58 & 0.51 & 0.51 & Zoo & NP \\
\hline & Eugenia cereja D. Legrand & 21 & 1.96 & 0.68 & 1.50 & Zoo & NP \\
\hline & Eugenia excelsa Cambess. & 2 & 0.23 & 0.03 & 0.15 & Zoo & NP \\
\hline & Eugenia fusca O. Berg & 20 & 1.85 & 0.79 & 1.47 & $\mathrm{NC}$ & $\mathrm{NC}$ \\
\hline & Eugenia linguaeformis $\mathrm{O}$. Berg & 6 & 0.69 & 0.37 & 0.53 & $\mathrm{NC}$ & $\mathrm{NC}$ \\
\hline & Eugenia magnibracteolata Mattos e D. Legrand & 6 & 0.69 & 1.20 & 0.81 & Zoo & NP \\
\hline & Eugenia melanogyna (D. Legrand) Sobral & 1 & 0.12 & 0.01 & 0.07 & Zoo & NP \\
\hline & Eugenia monosperma Vell. & 2 & 0.23 & 0.05 & 0.15 & Zoo & $\mathrm{NC}$ \\
\hline & Eugenia mosenii (Kausel) Sobral & 2 & 0.23 & 0.03 & 0.15 & Zoo & NP \\
\hline & Eugenia multicostata D. Legrand & 2 & 0.23 & 0.89 & 0.43 & Zoo & NP \\
\hline & Eugenia oblongata O. Berg & 20 & 1.96 & 1.75 & 1.83 & Zoo & NP \\
\hline & Eugenia prasina $\mathrm{O}$. Berg & 15 & 1.62 & 0.41 & 1.14 & Zoo & NP \\
\hline & Eugenia subavenia $\mathrm{O}$. Berg & 3 & 0.35 & 0.05 & 0.22 & Zoo & $\mathrm{P}$ \\
\hline & Eugenia sp.1 & 3 & 0.35 & 0.44 & 0.35 & $\mathrm{NC}$ & $\mathrm{NC}$ \\
\hline & Eugenia sp.2 & 7 & 0.69 & 0.56 & 0.63 & $\mathrm{NC}$ & $\mathrm{NC}$ \\
\hline & Eugenia sp.3 & 3 & 0.12 & 0.28 & 0.22 & $\mathrm{NC}$ & $\mathrm{NC}$ \\
\hline & Marlierea obscura O. Berg & 28 & 2.59 & 2.93 & 2.73 & Zoo & NP \\
\hline & Marlierea racemosa (Vell.) Kiaersk. & 2 & 0.23 & 0.14 & 0.18 & Zoo & NP \\
\hline & Marlierea riedeliana (O.Berg) D. Legrand & 2 & 0.23 & 0.09 & 0.17 & Zoo & $\mathrm{NC}$ \\
\hline & Marlierea silvatica (Gardner) Kiaersk. & 1 & 0.12 & 0.37 & 0.19 & Zoo & NP \\
\hline & Marlierea sp. & 2 & 0.23 & 0.02 & 0.14 & $\mathrm{NC}$ & NC \\
\hline & Myrcia insigniflora M.F.Santos & 7 & 0.81 & 0.88 & 0.77 & $\mathrm{NC}$ & NC \\
\hline & Myrcia neoblanchetiana E.Lucas \& Sobral & 6 & 0.58 & 0.25 & 0.45 & $\mathrm{NC}$ & $\mathrm{NC}$ \\
\hline & Myrcia pubipetala Miq. & 1 & 0.12 & 0.16 & 0.12 & Zoo & NP \\
\hline & Myrcia spectabilis DC. & 12 & 1.15 & 0.24 & 0.82 & Zoo & NP \\
\hline & Myrcia strigipes Mart.. & 22 & 2.31 & 1.48 & 1.95 & Zoo & NP \\
\hline & Myrciaria floribunda (H. West ex Willd.) O. Berg & 11 & 1.04 & 0.9 & 0.97 & Zoo & NP \\
\hline Nyctaginaceae & Guapira opposita (Vell.) Reitz & 34 & 3.23 & 4.08 & 3.48 & Zoo & NP \\
\hline Ochnaceae & Ouratea parviflora Baill. & 4 & 0.35 & 0.05 & 0.25 & Zoo & NP \\
\hline Peraceae & Pera glabrata (Schott) Poepp. ex Baill. & 2 & 0.23 & 0.12 & 0.18 & Zoo & $\mathrm{P}$ \\
\hline Phyllantaceae & Hyeronima alchorneoides Allemão & 8 & 0.81 & 3.03 & 1.52 & Zoo & NP \\
\hline Piperaceae & Piper cf. cernunn A.DC. & 4 & 0.46 & 0.05 & 0.29 & Zoo & $\mathrm{P}$ \\
\hline Polygonaceae & Ruprechtia laxiflora Meisn. & 1 & 0.12 & 0.69 & 0.30 & Ane & NP \\
\hline Primulaceae & Stylogyne lhotzkyana (A.DC.) Mez. & 2 & 0.23 & 0.05 & 0.15 & Zoo & NP \\
\hline Proteaceae & Roupala montana var. brasiliensis (Klotzsch) & 1 & 0.12 & 0.27 & 0.16 & Ane & NP \\
\hline Quiinaceae & Quiina glaziovii Engl. & 3 & 0.35 & 0.23 & 0.28 & Zoo & $\mathrm{P}$ \\
\hline Rosaceae & Prunus Myrtifolia (L.) Urb. & 4 & 0.46 & 0.6 & 0.47 & Zoo & NP \\
\hline \multirow{11}{*}{ Rubiaceae } & Bathysa australis (A. St.-Hil.) Benth e Hook. f. & 6 & 0.69 & 0.61 & 0.61 & Aut & NP \\
\hline & Bathysa mendoncaei K. Schum. & 68 & 5.20 & 2.77 & 4.67 & Aut & NP \\
\hline & Coussarea accedens Mull. Arg. & 34 & 3.23 & 1.15 & 2.47 & Zoo & $\mathrm{P}$ \\
\hline & Coussarea meridionalis (Vell.) Müll. Arg. & 73 & 5.08 & 2.08 & 4.56 & Zoo & NP \\
\hline & Faramea picinguabae M. Gomes & 7 & 0.81 & 0.07 & 0.50 & $\mathrm{NC}$ & $\mathrm{NC}$ \\
\hline & Ixora gardneriana Benth. & 1 & 0.12 & 0.07 & 0.09 & Zoo & NP \\
\hline & Psycotria mapoureoides DC. & 2 & 0.23 & 0.09 & 0.17 & Zoo & NP \\
\hline & Rudgea vellerea Müll. Arg. & 3 & 0.35 & 0.12 & 0.24 & Zoo & NP \\
\hline & Rustia formosa (Cham. e Schltdl. ex DC.) Klotzsch & 28 & 2.19 & 2.71 & 2.47 & Aut & $\mathrm{P}$ \\
\hline & Rubiaceae 1 & 1 & 0.12 & 0.06 & 0.09 & $\mathrm{NC}$ & $\mathrm{NC}$ \\
\hline & Rubiaceae 2 & 1 & 0.12 & 0.07 & 0.09 & $\mathrm{NC}$ & $\mathrm{NC}$ \\
\hline Salicaceae & Casearia sp. & 1 & 0.12 & 0.02 & 0.07 & $\mathrm{NC}$ & $\mathrm{NC}$ \\
\hline \multirow[t]{4}{*}{ Sapindaceae } & Cupania furfuracea Radkl. & 1 & 0.12 & 0.04 & 0.08 & Zoo & NP \\
\hline & Cupania oblongifolia Mart. & 16 & 1.27 & 4.04 & 2.25 & Zoo & NP \\
\hline & Matayba sp. & 1 & 0.12 & 0.02 & 0.07 & $\mathrm{NC}$ & $\mathrm{NC}$ \\
\hline & Sapindaceae 1 & 1 & 0.12 & 0.06 & 0.09 & $\mathrm{NC}$ & $\mathrm{NC}$ \\
\hline \multirow[t]{6}{*}{ Sapotaceae } & Chrysophyllum cf. paranaense T.D. Penn. & 1 & 0.12 & 0.02 & 0.08 & Zoo & $\mathrm{NC}$ \\
\hline & Chrysophyllum flexиosum Mart. & 30 & 3.12 & 1.41 & 2.40 & Zoo & NP \\
\hline & Chrysophyllum viride Mart. e Eichler & 2 & 0.23 & 2.74 & 1.05 & Zoo & NP \\
\hline & Ecclinusa ramiflora Mart. & 5 & 0.58 & 1.45 & 0.82 & Zoo & NP \\
\hline & Manilkara subsericea (Mart.) Dubard & 2 & 0.23 & 0.32 & 0.24 & Zoo & NP \\
\hline & Pouteria venosa (Mart.) Baehni & 4 & 0.46 & 0.25 & 0.36 & Zoo & NP \\
\hline Siparunaceae & Siparuna guianensis Aublet & 2 & 0.23 & 0.01 & 0.14 & Zoo & $\mathrm{P}$ \\
\hline \multirow[t]{2}{*}{ Urticaceae } & Cecropia glaziovi Snethl. & 4 & 0.35 & 0.48 & 0.39 & Zoo & $\mathrm{P}$ \\
\hline & Pourouma guianensis Aubl. & 2 & 0.23 & 0.46 & 0.29 & Zoo & $\mathrm{P}$ \\
\hline Verbenaceae & Citharexylum myrianthum Cham. & 1 & 0.12 & 0.12 & 0.11 & Zoo & $\mathrm{P}$ \\
\hline \multirow[t]{3}{*}{ Indeterminate } & Indeterminate 1 & 4 & 0.12 & 1.61 & 0.61 & $\mathrm{NC}$ & $\mathrm{NC}$ \\
\hline & Indeterminate 2 & 1 & 0.48 & 0.32 & 0.37 & $\mathrm{NC}$ & $\mathrm{NC}$ \\
\hline & Indeterminate 3 & 1 & 0.12 & 0.33 & 0.18 & $\mathrm{NC}$ & $\mathrm{NC}$ \\
\hline
\end{tabular}


The families with the highest species richness were Myrtaceae (32 species), Fabaceae (12), Rubiaceae (11), Euphorbiaceae (7), and Lauraceae (6) which together comprised $49.6 \%$ of the individuals surveyed. In the other hand, ten families just recorded one or two individuals. The highest values of importance (VI) were recorded for Myrtaceae (19.39\%), Rubiaceae (15.9\%), and Arecaceae $(9.3 \%)$.

Among the species, Euterpe edulis (IV $=6.9 \%)$ was the most important, followed by Mollinedia schottiana (5.9\%) and Bathysa mendoncaei (4.7\%). The Euterpe edulis was the most abundant species with 124 individuals (11.1\% of the total), followed by Mollinedia schottiana (8.3\%) and Coussarea meridionalis (6.5\%), which together comprised 290 individuals surveyed. While 43 species were represented by only one individual. Regarding the species accumulation curve, our study presented sampling sufficiency (Figure 2).

In the sampled plot, we found $23.01 \mathrm{~m}^{2}$ of basal area occupied by living individuals and $2.23 \mathrm{~m}^{2}$ occupied by stems deaths. The tree community presented $5.3 \%$ of the branched individuals, $7.9 \mathrm{~m}$ of mean height (standard deviation $=3.5$ ), with minimum and maximum values of 1.8 and $25 \mathrm{~m}$ respectively, and $12.7 \mathrm{~cm}$ of mean diameter (standard deviation $=9.9$ ), with minimum and maximum values of 4.5 and $87.5 \mathrm{~cm}$, respectively.

For the ecological groups of dispersal syndrome, zoochory was predominant, involving $87 \%$ of the species and $76.3 \%$ of the classified individuals, while anemochory and autochory were restricted to few species (Table 2). The anemochory group presented the higher mean value of basal area and, on the other hand, the smaller abundance among the groups considered for dispersal syndromes.

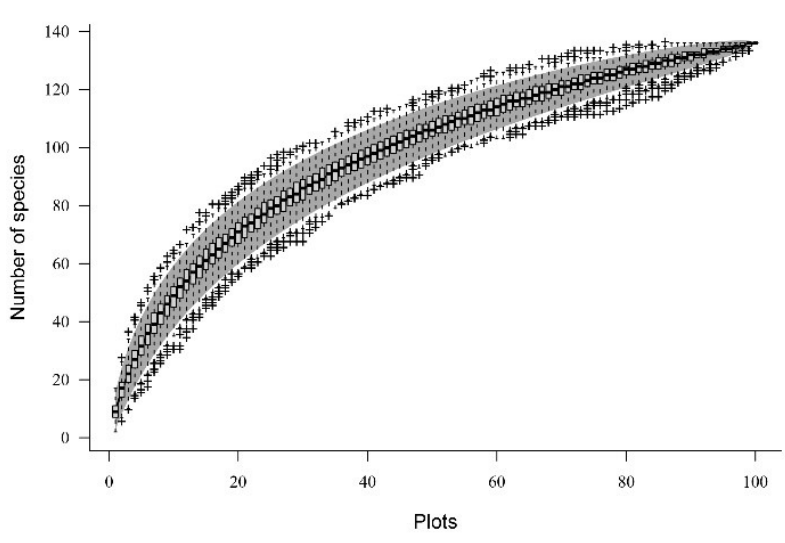

Figure 2. Species accumulated curve generated to tree community surveyed in the Atlantic Rainforest of Serra do Mar State Park, Ubatuba, Brazil.

Figura 2. Curva de acumulação gerada para a comunidade arbórea levantada na Floresta Pluvial Atlântica do Parque Estadual da Serra do Mar, Ubatuba, Brasil.

For the ecological groups of successional classes, the group of non-pioneers concerned $71.6 \%$ of species and $74.5 \%$ of individuals classified in the community sampled. However, we found the higher mean values of basal area and height for the pioneer's group (Table 2). Regarding height, some individuals of pioneer species stood out in the community with a maximum height greater than 23 meters, such as Eryotheca penthaphylla and Mabea piriri, raising the mean value of this attribute in the pioneer group.

Table 2. Structural parameters and diversity descriptors for different ecological groups in a tree community of in Atlantic Rainforest of Serra do Mar State Park, São Paulo, Brazil. Abd. - Abundance; N. spp. - Number of species; N. fam. - Number of families; Ab. Freq. Absolutefrequency; Ab. Do. - AbsoluteDominance.

Tabela 2. Parâmetro estruturais e descritores de diversidade para os diferentes grupos ecológicos de uma comunidade arbórea da Floresta Atlântica Estadual da Serra do Mar, São Paulo, Brasil. Abd. - Abundância; N. spp. - Número de espécies; N. fam.- Número de famílias; Ab. Freq. - Frequência absoluta; Ab.Do. - Dominância Absoluta.

\begin{tabular}{cccccccc}
\hline Ecological group & Abd. & N. spp. & N. fam. & $\begin{array}{c}\text { Ab. Freq. } \\
(\%)\end{array}$ & $\begin{array}{c}\text { Ab. Do. } \\
\left(\mathrm{m}^{2}\right)\end{array}$ & $\begin{array}{c}\text { Mean values } \\
\text { of height }(\mathrm{m})\end{array}$ & $\begin{array}{c}\text { Mean values of } \\
\text { basal area }\left(\mathrm{m}^{2}\right)\end{array}$ \\
\hline Anemochory & 57 & 9 & 7 & 32 & 1.7 & 7.78 & 0.30 \\
Autochory & 119 & 5 & 2 & 64 & 1.8 & 7.81 & 0.15 \\
Zoochory & 859 & 93 & 32 & 99 & 17.4 & 7.88 & 0.20 \\
Pioneer & 190 & 29 & 16 & 75 & 4.5 & 8.12 & 0.24 \\
Non-Pioneer & 840 & 75 & 28 & 99 & 16.3 & 7.86 & 0.19 \\
Community & 1.120 & 131 & 40 & 100 & 27.5 & 7.92 & 0.20 \\
\hline
\end{tabular}

\section{DISCUSSION}

The richest families of tree species in this survey were similar to several other studies carried out in the Atlantic Rainforest (CARVALHO et al., 2006; JOLY et al., 2012; KURTZ; ARAÚJO, 2000; MEDEIROS; AIDAR 2011; MARCHIORI et al., 2016; PADGURSCHI et al., 2011), and the species richness (131) was close to that registered in other surveys of the same vegetation type (ASSIS et al., 2011; PRATA et al., 2011). We found most individuals collected belonging to few species, which provided a great contribution to the richness observed. These results support the idea of the richness based mainly on species that occur with one or a few individuals in tropical forests (HUSTON, 1973; RABOSKY; HURLBERT, 2015). Regarding the species, we emphasize that Euterpe edulis, Mollinedia schottiana and Coussarea meridionalis were the highest abundant species of this survey, likewise found in other studies in Lowland Atlantic Rainforest (CAMPOS et al., 2011; PRATA et al., 2011; SANCHEZ et al., 2013).

In the study area, Myrtaceae and Fabaceae were the richest families, comprising a third of the total of identified species and presenting a proportional individuals distribution among its species. On the other hand, we found the ten most abundant species comprising around about $50 \%$ of individuals.

Overall, we have found a similar species composition to other studies carried out on preserved areas of this phytogeographic domain, and the sampling of large populations of threatened species, such as Euterpe edulis and Virola bicuhyba mainly, highlights the importance of new floristic and phytosociological surveys to understanding the conservation and species distribution of tree communities with high diversity. 
Our results indicate the most important families and species that are constant in preserved communities in the areas of the southeast Atlantic Rainforest (ASSIS et al. 2011; CARVALHO et al. 2007), and the tree diversity were similar of the majority surveys carried out in preserved communities of the Atlantic Rainforest (JOLY et al., 2012; KURTZ; ARAÚJO, 2000; SANCHEZ et al., 2013). Regarding the community structure, although the proportion of the number and basal area occupied by standing dead stems were higher in comparison with other communities surveyed in the same study area, we found consistent phytosociological parameters with these others tree communities (see JOLY et al. 2012).

Among the dispersal syndromes, we found that the distribution of individuals and species were classified following the distribution patterns of the Tropical Rainforest, in which the zoochoric species composed more than $70 \%$ of the species (CARVALHO, 2010; KINOSHITA et al., 2006; PENHALBER; MANTOVANI, 1997; PIVELLO et al., 2006). This result supports the idea that tropical forest tree diversity is mainly based on the group of zoochoric species, and its predominance would be related to the advanced preservation state and age of the community (LIEBSCH et al., 2008).

The species distribution among dispersal syndromes presents a variation trend in relation to the intensity of the dry season and the pluviometric regimes, in which the number of zoochoric species increases, in tree communities, from dry to humid regions (GENTRY, 1983). In addition, the number of zoochoric species is usually higher in preserved tropical forests, compared to communities located in disturbed areas or in initial stages of succession (BROWN; LUGO, 1990; CARVALHO 2010; NASCIMENTO et al., 2014).

We found a predominance of non-pioneer species among the succession classes, but the pioneer species presented a great contribution in the diversity of the tree community, involving about $30 \%$ of the species classified. The group of pioneers is a fundamental component in the tree community dynamic, and the richness of this group may be related to the presence of canopy gaps, allowing the establishment and maintenance of these populations, which are an essential part of forest dynamics (DENSLOW, 1980; TABARELLI et al., 2012).

Also, we found a preeminently mean value of height and basal area of pioneer species in the sampled community, but they occurred with a smaller abundance. This ecological group, whose main characteristic is its rapid growth in diameter and height, contributes to the structure maintenance of arboreal communities in the tropical rainforest (WHITMORE, 1989).

In general, mature forests tend to present a variation in their structure and species richness, which differ according to the successional stage of the community (HUBBEL et al., 1999; LIEBERMAN et al., 1990). However, higher values for basal area and species richness have been related to tree communities in preserved areas (BORÉM; OLIVEIRAFILHO, 2002; JOLY et al., 2012; KURTZ; ARAÚJO, 2000). Thus, the results of structure, tree diversity and mainly the species distribution among ecological groups suggest an advanced successional stage of the studied tree community. Finally, our study brings an important dataset and results about very threatened vegetation, corroborating the importance of tree communities' surveys to improve the understanding of conservation and species composition in areas with high diversity.

\section{CONCLUSIONS}

In a nutshell, we found a similar floristic and structural composition of the other communities surveyed in preserved areas of the same physiognomy, and the species contribution of different ecological groups in the structure of the studied community was congruent with other surveys in Tropical Rainforests.

In the tree community surveyed, Myrtaceae and Fabaceae were the richest families, and Euterpe edulis was the most abundant species.

We found a great population of some threatened species, and we corroborated the dominance of zoochoric and nonpioneer species in the preserved communities along a continuum of Atlantic Rainforest.

The study design was corrected to describe the floristic composition, phytosociological structure and ecological groups of a tree community in a poorly known physiognomy of the Atlantic Rainforest.

\section{ACKNOWLEDGEMENTS}

We thank the São Paulo Research Foundation (FAPESP) for financial support, and CNPq for the scholarship awarded to the first author (process number 141781/2016-5). We also thank all researchers that collaborated in the collection and identification of the botanical material, Henrique Lauand Ribeiro for helping on maps preparation, as well as the manager and staff of Serra do Mar State Park.

\section{REFERÊNCIAS}

APG IV; CHASE M. W.; CHRISTENHUSZ, M. J. M.; FAY, M. F.; BYNG, J. W.; JUDD, W. S.; SOLTIS, D. E.; MABBERLEY, D. J.; SENNIKOV, A. N.; SOLTIS, P. S.; STEVENS; P. F. An update of the Angiosperm Phylogeny Group classification for the orders and families of flowering plants: APG IV. Botanical Journal of the Linnean Society, v. 181, n. 1, p. 1-20, 2016.

ASSIS, M. A.; PRATA, E. M. B.; PEDRONI, F.; SANCHEZ, M.; EISENLOHR, P. V.; MARTINS, F. R.; SANTOS, F. A. M. DOS; TAMASHIRO, J. Y.; ALVES, L. F.; VIEIRA, S. A.; PICCOLO, M. DE C.; MARTINS, S. C.; CAMARGO, P. B. DE; CARMO, J. B. DO; SIMÕES, E.; MARTINELLI, L. A.; JOLY, C. A. Florestas de restinga e de terras baixas na planície costeira do sudeste do Brasil: vegetação e heterogeneidade ambiental. Biota Neotropica, Campinas, v. 11, n. 2, p. 103-121, 2011. DOI: http://dx.doi.org/10.1590/S1676-06032011000200012

BFG. Growing knowledge: an overview of Seed Plant diversity in Brazil. Rodriguésia v. 66, p.1085-1113, 2015.

BORÉM, R. A. T.; OLIVEIRA-FILHO, A.T. Fitossociologia do estrato arbóreo em uma topossequência alterada de Mata Atlântica, no município de Silva Jardim - RJ, Brasil. Revista Árvore, Viçosa, v. 26, n. 6, p. 27-742, 2002. DOI: http://dx.doi.org/10.1590/S0100-67622002000600009

BROWN, S.; LUGO, A. E. Tropical secondary forests. Journal of Tropical Ecology, v.6, p.1-32, 1990.

CAMPOS, M. C. R. de; TAMASHIRO, J. Y.; ASSIS, M. A.; JOLY, C. A. Florística e fitossociologia do componente arbóreo da transição Floresta Ombrófila Densa das Terras Baixas - Floresta Ombrófila Densa Submontana do Núcleo 
Picinguaba/PESM, Ubatuba, sudeste do Brasil. Biota Neotropica, Campinas, v. 11, n. 2, p. 301-312, 2011. DOI: http://dx.doi.org/10.1590/S1676-06032011000200030

CARVALHO, F. A.; NASCIMENTO, M. T.; BRAGA, J. M. A. Composição e riqueza florística do componente arbóreo da Floresta Atlântica submontana na região de Imbaú, Município de Silva Jardim, RJ. Acta Botanica Brasilica, Porto Alegre, v. 20, n. 3, p. 727-740, 2006. DOI: http://dx.doi.org/10.1590/S0102-33062006000300022

CARVALHO, F. A.; NASCIMENTO, M. T.; BRAGA, M. A. Estrutura e composição florística do estrato arbóreo de um remanescente de Mata Atlântica submontana no município de Rio Bonito, RJ, Brasil (Mata Rio Vermelho). Revista Árvore, Viçosa, v. 31, n. 4, 2007. DOI: http://dx.doi.org/10.1590/S0100-67622007000400017

CARVALHO, F. A. Síndromes de dispersão de espécies arbóreas de florestas ombrófilas submontanas do estado do Rio de Janeiro. Revista Árvore, Viçosa, v. 34, n. 6, p. 1017-1023, 2010. DOI: http://dx.doi.org/10.1590/S010067622010000600007

CATHARINO, E. L. M.; BERNACCI, L. C.; FRANCO, G. A. D. C.; DURIGAN, G.; METZGER, J. P. Aspectos da composição e diversidade do componente arbóreo das florestas da Reserva Florestal do Morro Grande, Cotia, SP. Biota Neotropica, Campinas, v. 6, n. 2, p. 1-18, 2006.

DENSLOW, J. S. Gap Partitioning among Tropical Rainforest Trees. Biotropica, Washington, v. 12, n. 2, p. 47-55, 1980. DOI: https://dx.doi.org/10.2307/2388156

EISENLOHR, P. V.; DE OLIVEIRA-FILHO, A. T. Revisiting Patterns of Tree Species Composition and their Driving Forces in the Atlantic Forests of Southeastern Brazil. Biotropica, Washington, v. 47, n. 6, p. 689-701, 2015. DOI: https://doi.org/10.1111/btp.12254

FIASCHI, P.; PIRANI, J. R. Review of plant biogeographic studies in Brazil. Journal of Systematics and Evolution, v. 47, n. 5, p. 477-496, 2009. DOI: https://doi.org/10.1111/j.1759-6831.2009.00046.x

GENTRY, A. H. Dispersal ecology and diversity in neotropical forest communities. Sonderbände Naturwissenschaftlichen Vereins im Hamburg, v. 7, p.303-314, 1983.

GRESSlER, E.; PIZO, M. A.; MOREllatO, L. P. C. Polinização e dispersão de sementes em Myrtaceae do Brasil. Revista Brasileira de Botânica, São Paulo, v. 29 , n. 4, p. 509-530, 2006. DOI: http://dx.doi.org/10.1590/S0100-84042006000400002

HUBBELL, S. P.; FOSTER, R. B.; BRIEN, S. T. O.; HARMS, K. E.; CONDIT, R.; WECHSLER, B.; WRIGHT, S. J.; LOO DE LAO, S. Light-gap disturbances, recruitment limitation, and tree diversity in a neotropical forest. Science, Washington, v. 283, n. 5401, p. 554-557, 1999. DOI: https://dx.doi.org/10.1126/science.283.5401.554

HUSTON, M. et al. A general hypothesis of species diversity. The American Naturalist, Chicago v. 113, n. 1, p. 81101, 1979. DOI: https://doi.org/10.1086/283366

IBGE_Instituto Brasileiro de Geografia e Estatística. Manual técnico da vegetação brasileira. Brasília: Manuais Técnicos em Geociências, n. 1, 2012.

JOLY, C. A.; ASSIS, M. A.; BERNACCI, L. C.; TAMASHIRO, J. Y.; CAMPOS, M. C. R DE, GOMES, J. A. M. A.; LACERDA, M. S., SANTOS, F. A. M. DOS, PEDRONI, F., PEREIRA, L. DE S., PADGURSCHI, M. DE C. G., PRATA, E. M. B.; RAMOS, R.; TORRES, R.
B.; ROCHELLE, A.; MARTINS, F. R.; ALVES, L. F.; VIEIRA, S. A.; MARTINELLI, L. A.; CAMARGO, P. B. DE; AIDAR, M. P. M.; EISENLOHR, P. V.; SIMÕES, E.; VILLANI, J. P.; BELINELLO, R. Floristic and phytosociology in permanent plots of the Atlantic Rainforest along an altitudinal gradient in southeastern Brazil. Biota Neotropica, Campinas, v. 12, n. 1, p. 123145, 2012. DOI: http://dx.doi.org/10.1590/S167606032012000100012

JOLY, C.A. \& MARTINELLI, L.A. 2008. Composição florística, estrutura e funcionamento da Floresta Ombrófila Densa dos Núcleos Picinguaba e Santa Virgínia do Parque Estadual da Serra do Mar, Estado de São Paulo, Brasil. $3^{\circ}$ Relatório do Projeto Temático Biota Gradiente Funcional. Processo FAPESP 03/12595-7.

KINOSHITA, L. S.; TORRES, R. B.; FORNI-MARTINS, E. R.; SPINELLI, T.; AHN, Y. J.; CONSTÂNCIO, S. S. Composição florística e síndromes de polinização e de dispersão da mata do Sítio São Francisco, Campinas, SP, Brasil. Acta Botanica Brasilica, Porto Alegre, v. 20, n. 2, p. 313-327, 2006. DOI: http://dx.doi.org/10.1590/S010233062006000200007

KURTZ, B. C.; ARAÚJO, D. S. D. Composição florística e estrutura do componente arbóreo de um trecho de Mata Atlântica na Estação Ecológica Estadual. Rodriguésia, Rio de Janeiro, v. 51, n. 21, p. 69-112, 2000. DOI: http://dx.doi.org/10.1590/2175-7860200051787903

LAURANCE, W. F. Conserving the hottest of the hotspots. Biological Conservation, Essex, v. 142, n. 6, p. 1137, 2009. DOI: https://doi.org/10.1016/j.biocon.2008.10.011

LEIGH, E. G.; DAVIDAR, P.; DICK, C. W.; TERBORGH, J.; PUYRAVAUD, J. P.; TER STEEGE, H.; WRIGHT, S. J. Why do some tropical forests have so many species of trees?. Biotropica, v. 36, n. 4, p.447-473, 2004. DOI: https://doi.org/10.1111/j.1744-7429.2004.tb00342.x

LIEBERMAN, D; HARTSHORN, G.S.; LIEBERMAN, M.; PERALTA, R. Forest dynamics at La Selva Biological Station, 1969-1985. In: GENTRY, A.H., ed. Four neotropical rainforests. New Haven: Yale University Press, 1990. p.509-521.

LIEBSCH, D.; MARQUES, M. C. M.; GOLDENBERG, R. How long does the Atlantic Rain Forest take to recover after a disturbance? Changes in species composition and ecological features during secondary succession. Biological Conservation, Essex, v. 141, n. 6, p. 17171725, 2008.2 DOI: https://doi.org/10.1016/j.biocon.2008.04.013

MARCHIORI, N. M.; ROCHA, H. R. da; TAMASHIRO, J. Y.; AIDAR, M. P. M. Tree community composition and aboveground biomass in a secondary Atlantic forest, Serra do Mar state park, Sao Paulo, Brazil. Cerne, Lavras, v. 22, n. $4, \quad$ p. 501-514, 2016. DOI: http://dx.doi.org/10.1590/01047760201622042242

MARTINELLI, G.; MORAES, M. A (Org.). Livro vermelho da flora do Brasil. Brasília: Centro Nacional de Conservação da Flora, 2013. 1102 p.

MEDEIROS, M. C. M. P.; AIDAR, M. P. M. Structural variation and content of aboveground living biomass in an area of Atlantic Forest in the State of São Paulo, Brazil. Hoehnea, São Paulo, v. 38, n. 3, p. 413-428, 2011. DOI: http://dx.doi.org/10.1590/S2236-89062011000300004

MITTERMEIER, R. A; TURNER, W. R.; LARSEN, F. W.; BROOKS, T. M.; GASCON, C. Global Biodiversity 
Conservation: The Critical Role of Hotspots. In: ZACHOS, F. E.; HABEL, J. C (Ed.). Biodiversity Hotspots, 2011. p. 3-22.

MORELLATO, L. P. C.; HADDAD, C. F. B. Introduction: The Brazilian Atlantic Forest. Biotropica, Washington, v. 32, n. 4b, p. 786-792, 2000. DOI: https://doi.org/10.1111/j.1744-7429.2000.tb00618.x

MORO, M. F.; MARTINS, F. R. de. Métodos de levantamento do componente arbóreo-arbustivo. In: Felfili-Fagg, J.M. Eisenlohr, P.V. Melo, M.M.R.F. Andrade, L.A. \& Meira Neto, J.A.A. (Eds.). Fitossociologia no Brasil: métodos e estudos de caso. Viçosa: Editora UFV, 2011. p. 174-212.

MUELLER-DOMBOIS, D.; ELLENBERG, D. Aims and methods of vegetation ecology. New York: Wiley, 1974 $547 \mathrm{p}$.

MYERS, N.; MITTERMEIER, R. A.; MITTERMEIER, C. G.; FONSECA, G. A. B. da; KENT. J. Biodiversity hotspots for conservation priorities. Nature, v. 403, n. 6772, p 853-8, 2000. DOI: https://doi.org/10.1038/35002501

NASCIMENTO, L. M. do; SAMPAIO, E. V. de S. B.; RODAL, M. J. N.; LINS-E-SILVA, A. C. B. Secondary succession in a fragmented Atlantic Forest landscape: evidence of structural and diversity convergence along a chronosequence. Journal of forest research, v. 19, n. 6, p. 501-513, 2014. DOI: https://doi.org/10.1007/s10310014-0441-6

OLIVEIRA-FILHO, A. T.; FONTES, M. A. L. Patterns of Floristic Differentiation among Atlantic Forests in Southeastern Brazil and the Influence of Climate. Biotropica, Washington, v. 32, n. 4b, p. 793-810, 2000. DOI: https://doi.org/10.1111/j.1744-7429.2000.tb00619.x

PADGURSCHI, M. C. G.; PEREIRA, L. de S.; TAMASHIRO, J. Y.; JOLY, C. A. Composição e similaridade florística entre duas áreas de Floresta Atlântica Montana, São Paulo, Brasil. Biota Neotropica, Campinas, v. 11, n. 2, p. 139-152, 2011. DOI: http://dx.doi.org/10.1590/S1676-06032011000200014

PENHALBER, E. DE; MANTOVANI, W. Flowering and seed rain in a secondary forest in Sao Paulo, southeastern Brazil. Revista Brasileira de Botânica, São Paulo, v. 20, n. 2, p. 205-220, 1997.

PIVELLO, V. R.; PETENON, D.; JESUS, F. M. de; MEIRELLES, S. T.; VIDAL, M. M.; ALONSO, R. de A. S.; FRANCO, G. A. D. C.; METZGER, J. P. Chuva de sementes em fragmentos de Floresta Atlântica (São Paulo, SP, Brasil), sob diferentes situações de conectividade, estrutura florestal e proximidade da borda. Acta Botanica Brasilica, Porto Alegre, v. 20, n. 4, p. 845-859, 2006. DOI: http://dx.doi.org/10.1590/S0102-33062006000400010

PRATA, E. M. B.; ASSIS, M. A.; JOLY, C. A. Composição florística e estrutura da comunidade arbórea na transição da Floresta Ombrófila Densa das Terras Baixas - Floresta Ombrófila Densa Submontana do Núcleo Picinguaba/PESM, Ubatuba, sudeste do Brasil. Biota Neotropica, Campinas, v. 11, n. 2, p. 285-299, 2011. DOI: http://dx.doi.org/10.1590/S1676-06032011000200029

RABOSKY, D. L.; HURLBERT, A. H. Species Richness at Continental Scales Is Dominated by Ecological Limits. The American Naturalist, v. 185, n. 5, p. 572-583, 2015. DOI: https://doi.org/10.1086/680850

R DEVELOPMENT CORE. Team R: A Language and Environment for Statistical Computing, 2017.
Disponível em: https://www.r-project.org/. Accessed on 30 March 2017.

RIBEIRO, M. C.; METZGER, J. P.; MARTERSEN, A. C.; PONZONI, F. J.; HIROTA, M. M. The Brazilian Atlantic Forest: How much is left, and how is the remaining forest distributed? Implications for conservation. Biological conservation, Essex, v. 142, n. 6, p. 1141-1153, jun. 2009. DOI: https://doi.org/10.1016/j.biocon.2009.02.021

SAITER, F. Z.; EISENLOHR, P. V.; FRANÇA, G. S.; STEHMANN, J. R.; THOMAS, W. W.; OLIVEIRAFILHO, A. T. Floristic units and their predictors unveiled in part of the Atlantic Forest hotspot: Implications for conservation planning. Anais da Academia Brasileira de Ciências, Rio de Janeiro, v. 87, n. 4, p. 2031-2046, 2015. DOI: http://dx.doi.org/10.1590/0001-3765201520140132

SANCHEZ, M.; PEDRONI, F.; EISENLOHR, P. V.; OLIVEIRA-FILHO, A. T. Changes in tree community composition and structure of Atlantic rain forest on a slope of the Serra do Mar range, southeastern Brazil, from near sea level to $1000 \mathrm{~m}$ of altitude. Flora: Morphology, Distribution, Functional Ecology of Plants, v. 208, n. 3, p. 184-196, 2013. DOI: https://doi.org/10.1016/j.flora.2013.03.002

SÃO PAULO (Estado). Secretaria do Meio Ambiente. Resolução 08/2008. Diário Oficial do Estado. Publicado em 01 de fevereiro de 2008, Seção I, páginas 31 e 32. Imprensa Oficial do Estado, São Paulo, 2008. Disponível em: $<$ http://www.ibot.sp.gov.br/pesquisa_cientifica/restau racao_ecologica/resolucao_SMA08-31.1.2008.pdf>. Accessed on 31 January 2017.

TABARELLI, M.; MANTOVANI, W. A riqueza de espécies arbóreas na floresta atlântica de encosta no estado de São Paulo (Brasil). Revista Brasileira de Botânica, v. 22, n. 2, p. 217-223, $1999 . \quad$ DOI: http://dx.doi.org/10.1590/S0100-84041999000200012

TABARELLI, M.; PERES, C. A. Abiotic and vertebrate seed dispersal in the Brazilian Atlantic forest: Implications for forest regeneration. Biological Conservation, Essex, v. 106 , n. 2, p. 165-176, 2002. DOI: https://doi.org/10.1016/S0006-3207(01)00243-9

TABARELLI, M.; PERES, C. A.; MELO, F. P. L. The "few winners and many losers" paradigm revisited: Emerging prospects for tropical forest biodiversity. Biological Conservation, Essex, v. 155, p. 136-140, out. 2012. DOI: https://doi.org/10.1016/j.biocon.2012.06.020

TURNER, I. M. Species loss in fragments of tropical rain forest: a review of the evidence. Journal of Applied Ecology, Oxford, v. 33, n. 33, p. 200-209, 1996. DOI: https://dx.doi.org/10.2307/2404743

VAN DER PIJL, L. Principles of dispersal in higher plants. 3.ed. Springer-Verlag, Berlin, 1982, p. 978.

WHITMORE, T. C. Canopy gaps and the two major groups of forest trees. Ecology, Nova York, v. 70, n. 3, p. 536-538, 1989. DOI: https://doi.org/10.2307/1940195

WIJDEVEN, S. M. J.; KUZEE, M. E. Seed availability as a limiting factor in forest recovery processes in Costa Rica. Restoration Ecology, Malden, v. 8, n. 4, p. 414-424, 2000. DOI: https://doi.org/10.1046/j.1526100x.2000.80056.x 